\title{
Space-Time Multilevel Codes
}

\author{
Philippa A. Martin, David M. Rankin and Desmond P. Taylor \\ Department of Electrical and Computer Engineering, \\ University of Canterbury, Christchurch, New Zealand. \\ \{philippa_martin,dave_rankin\}@ ieee.org, taylor@elec.canterbury.ac.nz
}

\begin{abstract}
To date there has been little work done on multilevel codes for the space-time environment. In this paper we develop multi-dimensional space-time multilevel codes (ST-MLCs), which partition the $2 N_{t}$-dimensional signalling space that spans all $N_{t}$ transmit antennas. The partitioning is designed to reduce the complexity of detection/ decoding. We also develop a spacetime multistage decoder for the proposed ST-MLC. It allows the detection/ decoding complexity to be significantly reduced compared to a single level approach.
\end{abstract}

\section{INTRODUCTION}

To date there has been little research into using multilevel codes $(M L C s)$ in a space-time (multiple transmit antenna) environment. To our knowledge, the first reference to multilevel space-time codes (STCS) is Section III-H in [1]. In [1] the signal constellation ( $M$-QAM or $M$-PSK) ${ }^{1}$ is partitioned into $P$ levels using set partitioning [2] (and the idea of cosets), where each level uses a different encoder. The example in [1] uses binary partitions and simple length $N_{t}$ block codes on each level, where $N_{t}$ is the number of transmit antennas.

The space-time MLC presented in [3], [4] uses $P=$ $N_{t} \log _{2}(M)$ levels, where each level uses a binary partition of the 2-dimensional (2-D) constellation ( $M$-PSK or $M$-QAM) for a single transmit antenna [3], [4]. Thus, each component code in the MLC spans a single transmit antenna, although it is mentioned that a multi-dimensional (multi-D) mapping could be used [3]. The design strategy is based on capacity arguments [3] and equivalent channel capacities are used to specify the desired component code rates [3], [4].

Hybrid coded modulation (HCM) for the space-time environment is also proposed in [3]. It uses $N_{t}$ levels, where each level uses a bit interleaved coded modulation (BICM) scheme over $\log _{2}(M)$ bits and a single transmit antenna. Thus, each antenna uses a separate encoder and bit interleaver. It is similar to V-BLAST in some cases [3]. HCM provides similar performance to the MLCs presented in [3], but is simpler due to using fewer levels $\left(N_{t}\right.$ rather than $N_{t} \log _{2}(M)$ ) [3]. However, MLCs do not have to use binary partitions [5], as in [3], and so can also have fewer levels. Also bit interleaving can be added to any level of the MLC [6]. The decoding algorithms for the HCM and MLC schemes of [3] appear to be based on successive interference cancellation and multistage decoding.

In [7] (and several similar papers by the authors of [7]) MLCs are concatenated with orthogonal space-time block codes (OSTBCs).

\footnotetext{
${ }^{1} M$-ary quadrature amplitude modulation (M-QAM) and $M$-ary phase shift keying (M-PSK).
}

In this paper we design MLCs using multi-D partitioning of a $2 N_{t}$-dimensional $\left(2 N_{t}-D\right)$ real constellation or a $N_{t^{-}}$dimensional $\left(N_{t}-D\right)$ complex constellation. Binary and nonbinary partitions can be used on each level. In this work we will describe a $2 N_{t}$-D partitioning strategy based on [8], which we call partitioning by types. It allows each component code in the MLC to span all $N_{t}$ transmit antennas. Furthermore, no additional STC is used; instead we directly design space-time MLCs (ST-MLCs). Finally, we develop a space-time multistage decoder (ST-MSD) for the proposed ST-MLC. By carefully designing the partitioning for the ST-MLC we can reduce the computational complexity of the ST-MSD.

\section{SySTEM DESCRIPTION}

We consider space-time systems with $N_{t}$ transmit and $N_{r}$ receive antennas. The received vector can be written as

$$
\boldsymbol{r}^{t}=\boldsymbol{s}^{t} \boldsymbol{H}^{t}+\boldsymbol{n}^{t}
$$

where $t$ is the time index, $\boldsymbol{s}^{t}$ is the transmitted vector and $\boldsymbol{n}^{t}$ is the complex additive white Gaussian noise (AWGN) vector. $\boldsymbol{H}^{t}$ denotes the complex $N_{t} \times N_{r}$ channel matrix and $h_{i, j}^{t}$ is the element representing the subchannel from the $i^{\text {th }}$ transmit antenna to the $j^{t h}$ receive antenna. We assume that all subchannels are independent and that we have ideal channel state information at the receiver, but none at the transmitter.

In this paper we consider two flat Rayleigh fading channel models. The first is a correlated fading channel, where the speed of the fading is defined by the normalized fade rate, $f_{D} T, T$ being the symbol period. A $3^{\text {rd }}$ order Butterworth filter with cutoff frequency $f_{D}$ is used to generate the fading gains, $h_{i, j}^{t}$, for each subchannel. The second is an independent or ideally interleaved flat Rayleigh fading channel (as in [3]). In this case, the fading channel gains vary independently in each time slot and each $h_{i, j}^{t}$ is a complex Gaussian random variable with zero mean and unit variance. This approximates very fast fading or a system with sufficient interleaving.

The 1-D noise variance is defined as ${ }^{2}$ [10], [11], [12]

$$
\sigma_{n}^{2}=\frac{N_{t} E_{s}}{2 \log _{2}(M) \mathcal{R}_{\text {ecc }}^{\text {tot }} 10^{0.1 S N R}}
$$

where $S N R$ is the average signal to noise ratio $\left(E_{b} / N_{0}\right)$ in decibels $(d B), E_{s}$ is the average energy of a 2-D constellation point, $E_{b}$ is the average energy per data bit and $N_{0}$ is the

\footnotetext{
${ }^{2}$ This is equivalent to the definition in [9] when $N_{t}=N_{r}$.
} 


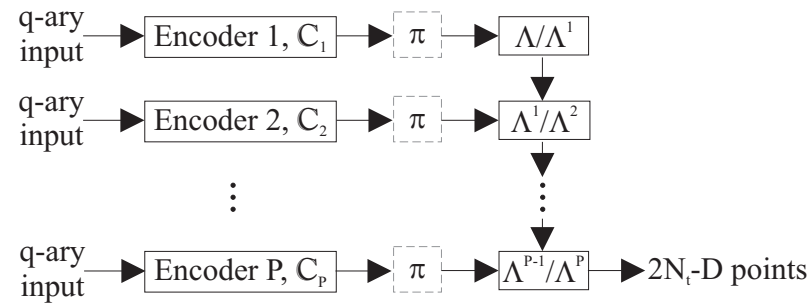

Fig. 1. Proposed $P$-level ST-MLC encoder with optional interleavers.

2-sided noise spectral density. The total rate of the error correction coding (over all levels) is defined as

$$
\mathcal{R}_{\text {ecc }}^{\text {tot }}=\frac{\sum_{p=1}^{P} k_{p} \log _{2}\left(q_{p}\right)}{\sum_{p=1}^{P} n_{p} \log _{2}\left(q_{p}\right)},
$$

where $k_{p}$ and $n_{p}$ are the number of $G F\left(q_{p}\right)$ data symbols and encoded symbols in the level $p$ component code, respectively. The rate of the ST-MLC is defined as $\mathcal{R}_{s t c}=\mathcal{R}_{\text {ecc }}^{\text {tot }} N_{t} 2-\mathrm{D}$ data constellation points transmitted per time slot. This allows for STC rates significantly greater than one.

\section{ST-MLC DESIGN}

Throughout we assume $r N_{r} \geq 4$, where $r$ is the rank of the code difference matrix of the ST-MLC. In [13] it was found that when $r N_{r} \geq 4$ the minimum Euclidean distance of the STC dominates performance. Therefore, we will design the ST-MLCs for large Euclidean distance.

The proposed $P$-level ST-MLC encoder structure is shown in Fig. 1. It includes optional bit or symbol interleaving on each level. Interleaving could also be added before the transmit antennas. We use the terminology of coset codes [14], [15]. We denote the partition chain by $\Lambda / \Lambda^{1} / \Lambda^{2} / \cdots / \Lambda^{P}$, where $P$ is the number of levels/ partitions used, $\Lambda$ is the overall $2 N_{t}$-D lattice/ constellation considered by the MLC and $\Lambda^{p}$ is the $p^{t h} 2 N_{t}$-D lattice/ constellation in the partition chain. Here we constrain the 2-D components of the $2 N_{t}$-D points to be elements of $M$-QAM, but other approaches could be used.

\section{A. Partitioning and Constellations}

One of the biggest issues with many non-orthogonal STCs is the computational complexity of detection/ decoding. The proposed ST-MLCs are non-orthogonal. Therefore, it is beneficial to design them (constellations, partitioning and component codes) with decoding in mind. To this end we use the multiD partitioning strategy of [8] to design the ST-MLCs and corresponding ST-MSD. We call this the partitioning by types design method. It is based on iteratively partitioning lower dimensional constituent lattices/ constellations [8]. As a result the $2 N_{t}$-D mapping can be done as a sequence of 2-D mappings rather than as a single $2 N_{t}$-D mapping. This allows the computational complexity of detection in the ST-MSD to be reduced.

The design of the partitions starts by considering the 2D constituent constellation, then higher dimensional constellations and partitions are constructed from it. We begin by choosing the desired (realizable) minimum squared Euclidean distance (MSED) between points in the subsets for the current level. Then the 2-D constellation is partitioned to give the desired MSED between points in the 2-D subsets. Consider the following 16-QAM constellation

\begin{tabular}{ll|ll}
$A$ & $B$ & $A$ & $B$ \\
$C$ & $D$ & $C$ & $D$ \\
\hline$A$ & $B$ & $A$ & $B$ \\
$C$ & $D$ & $C$ & $D$
\end{tabular}

and a desired MSED of $4 \delta_{0}^{2}$, where $\delta_{0}^{2}$ is the MSED between 16-QAM points. A 4-way partitioning [8] can be done in two stages. First divide the constellation into subsets $A \cup D$ and $B \cup C$, which gives MSED $2 \delta_{0}^{2}$. Second divide $A \cup D$ and $B \cup C$ into $A, B, C$ and $D$, which gives MSED $4 \delta_{0}^{2}$ as desired. This gives us four subsets of points, each called 2-D types, which are denoted $A, B, C$ and $D$. Note that this is also a four-way set partitioning [2] of 16-QAM.

Now we can create higher dimensional types by concatenating 2-D types [8]. For example, AABC is an 8-D type, which transmits a point from $\mathrm{A}$ in the first and second 2-D subsets, then a point from $\mathrm{B}$ and $\mathrm{C}$ in the third and fourth 2-D subsets, respectively. In our case each 2-D subset is transmitted from a different transmit antenna. The MSED of each 8-D type is that of the constituent 2-D types, in this case $4 \delta_{0}^{2}$. Many other kinds of 2-D mappings can be extended to $2 N_{t}$-D using this approach. For example, the diversity-based approaches of [11], [12], [16], [17] could be used (for $r N_{r}<4$ ).

\section{B. Component Codes}

As can be seen in Fig. 1 the component error correction code on each of the $P$ levels of the ST-MLC chooses a subset of $2 N_{t}$-D points to be transmitted. Collectively the $P$ component codes select a sequence of $2 N_{t}$-D points to be transmitted by the $N_{t}$ transmit antennas. Each $2 N_{t}$-D point is transmitted in a single time slot. Almost any coding scheme can be used as a component code in the ST-MLC [5], including concatenated codes or BICM. The ST-MLCs span $L$ time slots, where $L$ depends on the length and field size of the component codes, and the number, $P$, and size, $\left|\Lambda^{p-1} / \Lambda^{p}\right|$, of the partition levels. We define the length of the $q_{p}$-ary component code on level $p$ as $n_{p}=L \log _{q_{p}}\left(\left|\Lambda^{p-1} / \Lambda^{p}\right|\right) q_{p}$-ary symbols.

The component codes in the ST-MLC need to perform well at the desired rates, have feasible soft decision decoding complexity and good distance properties. MLCs tend to require component codes with a wide range of rates, as a result different classes of codes may be required on each level.

We choose the component code rates using the capacity design rule [18], [19]. We followed the approach in [3] to determine the capacity of each level in the partition chain. This enabled us to find the ideal channel coding rates which allow the overall channel capacity to be approached [18]. A variety of other rate design rules could be used such as those summarized in [18] (developed for the AWGN channel). 


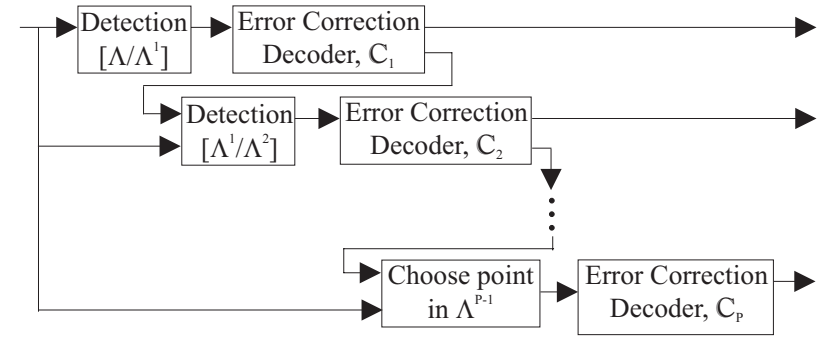

Fig. 2. Proposed ST-MSD for a P-level ST-MLC. If interleavers are used in the ST-MLC, then deinterleavers would need to be added to the ST-MSD.

\section{Detection and Decoding}

Decoding the composite MLC is usually prohibitively complex and so a multistage decoder is typically used. We now develop a ST-MSD for the proposed ST-MLCs. Its overall structure is shown in Fig. 2. The detection block on level $p$ calculates soft information for the level $p$ error correction decoder. We now describe the soft information calculation for each level in the ST-MSD. For simplicity of notation we assume binary component codes, however, the mathematics is easily extendable to the non-binary case.

Consider level $p$, which corresponds to partition $\Lambda^{p-1} / \Lambda^{p}$. The coset representatives can be labelled by $m_{p}=$ $\log _{2}\left(\left|\Lambda^{p-1} / \Lambda^{p}\right|\right)$ bits or by $\log _{q_{p}}\left(\left|\Lambda^{p-1} / \Lambda^{p}\right|\right) q_{p}$-ary symbols. The $i^{t h}$ bit labelling the level $p$ coset at time $t$ is denoted $b_{i t}^{p}$ and bits $\left(b_{1 t}^{p}, \cdots, b_{m_{p} t}^{p}\right)$ determine the transmitted level $p$ coset, indexed by $C_{p}$. We assume all $2 N_{t}$-D constellation points are equiprobable. The soft input to the binary error correction decoder corresponding to bit $b_{i t}^{p}$ can be written as

$$
\begin{aligned}
\lambda_{i t}^{p} & =\log \left(\frac{\operatorname{Pr}\left(b_{i t}^{p}=1 \mid \boldsymbol{r}^{t}, \boldsymbol{H}^{t}, \Phi_{p}\right)}{\operatorname{Pr}\left(b_{i t}^{p}=0 \mid \boldsymbol{r}^{t}, \boldsymbol{H}^{t}, \Phi_{p}\right)}\right) \\
& =\log \left(\frac{\sum_{\boldsymbol{s}^{1} \in\left\{\Lambda \mid b_{i t}^{p}=1, \Phi_{p}\right\}} \exp \left(-\frac{1}{2 \sigma_{n}^{2}}\left\|\boldsymbol{r}^{t}-\boldsymbol{H}^{t} \boldsymbol{s}^{1}\right\|^{2}\right)}{\sum_{\boldsymbol{s}^{0} \in\left\{\Lambda \mid b_{i t}^{p}=0, \Phi_{p}\right\}} \exp \left(-\frac{1}{2 \sigma_{n}^{2}}\left\|\boldsymbol{r}^{t}-\boldsymbol{H}^{t} \boldsymbol{s}^{0}\right\|^{2}\right)}\right)
\end{aligned}
$$

where $\Phi_{1}=\{\}$ (an empty set), $\Phi_{p}=\left(\hat{C}_{1}, \hat{C}_{2}, \cdots, \hat{C}_{p-1}\right)$ for $p>1$, and $\hat{C}_{l}$ is the index of the coset decision from level $l$. The max-log MAP approximation [20] of (5) is given by

$$
\lambda_{i t}^{p} \approx \frac{1}{2 \sigma_{n}^{2}}\left(\left\|\boldsymbol{r}^{t}-\boldsymbol{H}^{t} \boldsymbol{s}^{0, \max }\right\|^{2}-\left\|\boldsymbol{r}^{t}-\boldsymbol{H}^{t} \boldsymbol{s}^{1, \max }\right\|^{2}\right),
$$

where for $a \in\{0,1\}$

$$
\begin{aligned}
\boldsymbol{s}^{a, \max }= & \arg \left(\max _{\boldsymbol{s}^{a} \in\left\{\Lambda \mid b_{i t}^{p}=a, \Phi_{p}\right\}}\right. \\
& \left.\left(\exp \left(\frac{-1}{2 \sigma_{n}^{2}}\left\|\boldsymbol{r}^{t}-\boldsymbol{H}^{t} \boldsymbol{s}^{a}\right\|^{2}\right)\right)\right) .
\end{aligned}
$$

\section{EXAMPLE ST-MLC}

In this section we design a ST-MLC for $N_{t}=N_{r}=4$. Since $r N_{r} \geq 4$ [13] we design for large Euclidean distance.

\section{A. Partitioning}

We now design the partitioning by types for the STMLC. The 2-D, 4-D and 8-D types can be selected in a variety of different ways. Here for simplicity and in order to ensure good Euclidean distance we select the 8-D types using the $Z^{8} / E_{8} / R E_{8} / 2 E_{8} / 2 R E_{8}$ [14], [15] partition chain constrained to 16-QAM in each two dimensions [8]. We use a translated scaled subset of these lattices and sublattices. The following coset representatives are used for the partitions ${ }^{3}$

$$
\begin{aligned}
{\left[\Lambda / \Lambda^{1}\right] } & =2\left[b_{1 t}^{1}, b_{2 t}^{1}, b_{3 t}^{1}, b_{4 t}^{1}\right] \boldsymbol{G}_{1} \\
{\left[\Lambda^{1} / \Lambda^{2}\right] } & =2\left[b_{1 t}^{2}, b_{2 t}^{2}, b_{3 t}^{2}\right] \boldsymbol{G}_{2}-4\left[0, \cdots, 0, b_{4 t}^{2}\right] \\
{\left[\Lambda^{2} / \Lambda^{3}\right] } & =[2,2, \cdots, 2] b_{1 t}^{3}-4\left[b_{2 t}^{3}, b_{3 t}^{3}, b_{4 t}^{3}\right] \boldsymbol{G}_{3} \\
{\left[\Lambda^{3} / \Lambda^{4}\right] } & =4\left[b_{1 t}^{4}, b_{2 t}^{4}, b_{3 t}^{4}, b_{4 t}^{4}\right] \boldsymbol{G}_{4}+[1,1, \cdots, 1],
\end{aligned}
$$

where $m_{1}=m_{2}=m_{3}=m_{4}=4$,

$$
\begin{aligned}
\boldsymbol{G}_{1}= & {\left[\begin{array}{llllllll}
0 & 0 & 0 & 0 & 0 & 0 & 1 & 1 \\
0 & 0 & 0 & 0 & 0 & 1 & 0 & 1 \\
0 & 0 & 0 & 1 & 0 & 0 & 0 & 1 \\
0 & 0 & 0 & 0 & 0 & 0 & 0 & 1
\end{array}\right], } \\
\boldsymbol{G}_{4}= & {\left[\begin{array}{llllllll}
1 & 0 & 0 & 0 & 1 & 1 & 0 & 1 \\
0 & 1 & 0 & 0 & 0 & 1 & 1 & 1 \\
0 & 0 & 1 & 0 & 1 & 1 & 1 & 0 \\
0 & 0 & 0 & 1 & 1 & 0 & 1 & 1
\end{array}\right], }
\end{aligned}
$$

$\boldsymbol{G}_{2}$ is the last 3 rows of $\boldsymbol{G}_{4}$ and $\boldsymbol{G}_{3}$ is the first 3 rows of $\boldsymbol{G}_{1}$.

We shall create the 8-D types using the 2-D types (A, B, C and $\mathrm{D}$ ) in (4). These 2-D types and resulting 8-D types provide an MSED of $4 \delta_{0}^{2}$. There are $168 D$-types which collectively define coset $C_{1}=0$ for $\Lambda / \Lambda^{1}$, namely

$\begin{array}{llll}C B A A & A C A B & B D A C & D A A D \\ D C B A & B B B B & A A B C & C D B D \\ B A C A & D D C B & C C C C & A B C D \\ A D D A & C A D B & D B D C & B C D D .\end{array}$

The 16 8-D types for each of the other level 1 cosets can be found by adding the appropriate coset representative, (8), to the points corresponding to the 8 -D types in (13). This produces 16 new 8-D types.

We need to further partition the 2-D types in (4) to provide a MSED of $8 \delta_{0}^{2}$ for level 2. Each of the four 2-D types in (4) are split into two subsets to produce the eight 2-D types ( $E, F, G, H, I, J, K, L)$,

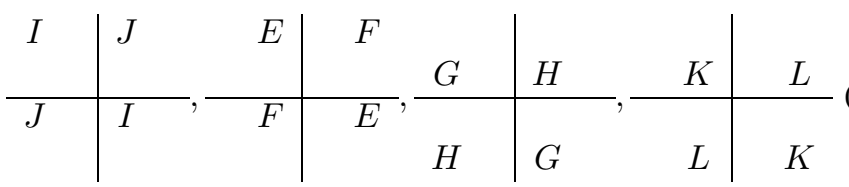

where $A=I \cup J, B=E \cup F, C=G \cup H$ and $D=K \cup L$. There are $168 D$-types which collectively define coset $\left(C_{1}=\right.$

\footnotetext{
${ }^{3}$ If the value of the coset representative added to an 8-D point is greater than 3 or smaller than -3 in any 1-D subset, then we subtract 8 or add 8 to the value, respectively, until it is in the set $\{ \pm 1, \pm 3\}$. This is basically a folding operation to ensure that we transmit an appropriate point within 16-QAM.
} 


$\begin{array}{rccl}\left.0, C_{2}=0\right) \text { for } \Lambda^{1} / \Lambda^{2}, \text { namely } & & \\ G G G G & G G H H & F F E E & F F F F \\ G H H G & G H G H & F E F E & F E E F \\ H H G G & H H H H & E F F E & E F E F \\ H G H G & H G G H & E E E E & E E F F\end{array}$

The 8-D types for other level 1 and 2 coset combinations are created by adding the corresponding coset representatives, (8) and (9), to the points defined by (15).

For the level 3 partition, the $2 D$-types in (14) are partitioned to give individual points. The partitioning for the $2 D$-types $E$, $F, G$ and $H$ is

\begin{tabular}{|c|c|}
\hline $\begin{array}{ll} & E_{1} \\
G_{1} & \\
\end{array}$ & $H_{2}{ }^{F_{2}}$ \\
\hline $\begin{array}{ll} & F_{1} \\
H_{1} & \end{array}$ & $\begin{array}{ll} & E_{2} \\
G_{2} & \end{array}$ \\
\hline
\end{tabular}

The $168 D$-types which collectively define coset $\left(C_{1}=\right.$ $\left.0, C_{2}=0, C_{3}=0\right)$ for $\Lambda^{2} / \Lambda^{3}$ are

$$
\begin{array}{cccc}
H_{2} G_{2} G_{1} H_{1} & H_{2} H_{2} H_{2} H_{2} & H_{2} G_{1} H_{1} G_{1} & H_{2} H_{1} G_{2} G_{2} \\
G_{2} H_{2} G_{2} H_{1} & G_{2} G_{2} H_{1} H_{2} & G_{2} H_{1} H_{2} G_{1} & G_{2} G_{1} G_{1} G_{2} \\
H_{1} H_{1} H_{1} H_{1} & H_{1} G_{1} G_{2} H_{2} & H_{1} H_{2} G_{1} G_{1} & H_{1} G_{2} H_{2} G_{2} \\
G_{1} G_{1} H_{2} H_{1} & G_{1} H_{1} G_{1} H_{2} & G_{1} G_{2} G_{2} G_{1} & G_{1} H_{2} H_{1} G_{2} .
\end{array}
$$

Now each 8-D type is in fact a single 8-D point. Again the 8-D types for other level 1,2 and 3 coset combinations are created by adding the corresponding coset representatives, (8), (9) and (10), to the points in (17). On the final level each coset is a single 8 -D type/ point.

\section{B. Component Codes}

In the present work we use binary irregular low density parity check ( $L D P C)$ component codes on each level, with rates chosen using the capacity rule [18], [19]. LDPC codes were chosen due to their excellent performance and simple iterative decoding algorithm (belief propagation). However, many other types of component codes could be used.

The LDPC codes used here are designed using the near optimal degree sequences for the AWGN channel based on techniques in [21]. Specifically, the codes are designed for maximum girth using a progressive edge growth algorithm [22], which ensures an upper triangular parity check matrix for linear time encoding. The decoder uses belief propagation [23] operating in the log-domain [20]. The maximum number of decoding iterations for the LDPC code is 100, although the LDPC decoder will terminate early if a codeword is found. A bit interleaver is used on each level after the LDPC encoder.

\section{Detection and Decoding}

The basic structure of the proposed ST-MSD is given in Fig. 2. On each level we use a bank of sphere decoders (SDs) for detection, with a separate SD for each coset. Each SD finds the best point in the coset, chosen according to the MSED from the received signal. Together the chosen points estimate a basic signal set [5], which is used to calculate soft information for the error correction decoder. The basic signal set consists of $\left|\Lambda^{p-1} / \Lambda^{p}\right|$ constellation points $\left(2 N_{t}-D\right)$, namely the best point from each coset, $\left[\Lambda^{p-1} / \Lambda^{p}\right]$. Soft information is calculated using (6). The SD for a given coset considers points from the coset which lie within a radius $\sqrt{C}$ of the received signal $^{4}$, where $C$ is a user defined positive constant [9], [24]. Alternatively, a bank of list SDs or a single large list SD [9], [11] could be used to calculate the soft information using (5).

Partitioning by types allows us to reduce the complexity of detection. Consider level 1, which has the 8-D types shown in (13) for coset $C_{1}=0$. All combinations of 2-D types are allowed over two antennas, but only a single 4-D type is allowed to be transmitted from the remaining two antennas (determined by the combination of 2-D types for the other two antennas). Thus, the SD considers all possible values in four dimensions and uses the 8-D types to define the allowed values in the other four dimensions. So, a maximum of 16 points needs to be considered by the level 1 detection algorithm for two of the transmit antennas and a maximum of four points for each of the other two antennas. The SD uses lookup tables to determine the 2-D points allowed to be considered for each transmit antenna. It orders the 2-D types/ constellation points according to reliability [25]. Specifically we use a modified version $^{5}$ of the SD in [11] (based on [9], [10], [25]). Likewise levels 2 and 3 can use the 8-D type information of (13) and (15) to reduce the computational complexity of the SD.

After detection and soft information calculation, the component decoder makes a hard decision on the transmitted coset, which is sent to the next level in the ST-MSD. This process continues until the last level, where the component decoder chooses a single $2 N_{t}$-D constellation point.

\section{Results}

In this section simulation results for the ST-MLC described in Section IV are given. Many other ST-MLCs can be developed using the proposed framework. We consider a 3-level ST-MLC designed based on the partition chain $E_{8} / R E_{8} / 2 E_{8} / 2 R E_{8}$. We use 3 rather than 4 levels as it is less complex and the design rate for the $Z^{8} / E_{8}$ component code was very low (near zero). The ST-MLC spans $L=1024$ time slots. The $(4096,1228),(4096,3072)$ and $(4096,3892)$ LDPC codes are used on level 1, 2 and 3, respectively. The overall code rate is $\mathcal{R}_{\text {ecc }}^{t o t}=0.5$ and the STC rate is $\mathcal{R}_{\text {stc }}=2$ data 16-QAM symbols per time slot. Therefore, 8 data bits are transmitted during each time slot. The bit error rate (BER) performance of this ST-MLC is shown in Fig. 3.

For comparison purposes $^{6}$ we consider a single level

\footnotetext{
${ }^{4}$ If no point is found by a SD, then the distance used to calculate the soft information is set to $C$. Alternatively, the SD could run again using a larger value of $C$. This is not considered here for complexity reasons.

${ }^{5}$ It is modified so that the 2-D types are restricted based on the 168 -D types associated with the coset.

${ }^{6}$ We will not compare the performance of the proposed ST-MLC to those of [3] as they use only $N_{t}=2$ or 3 and $N_{r}=1$ or 2 . We design for $r N_{r} \geq 4$, meaning their designs would need to provide $r=2$ (full rank) and use $N_{r}=2$. They consider 4-PSK, whereas we are interested in 16-QAM. In addition, a clear description of the decoding process is not given in [3].
} 


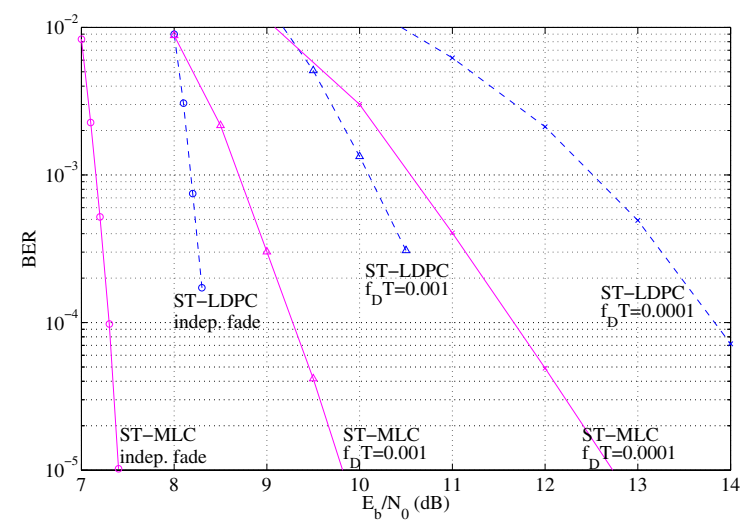

Fig. 3. BER of a rate $\mathcal{R}_{\text {stc }}=2$ ST-MLC and ST-LDPC code.

$(16384,8192)$ ST-LDPC code Gray mapped to 16-QAM across the $N_{t}=4$ transmit antennas and $L=1024$ time slots. A bit interleaver is used after encoding (not needed). It uses the soft output list SD of [9] with a list size of 512 (iterative decoding between the SD and error correction decoder is not considered here). The BER performance of this code is also shown in Fig. 3. As can be seen the performance of both codes is worse for slower fading channels.

The decoder of [9] was used for a rate $1 / 2$ binary Turbo code with 9216 data bits (and 18432 encoded bits) in [9]. They used $N_{t}=N_{r}=4$, an independent Rayleigh flat fading channel and 16-QAM. The Turbo code obtained a BER of $10^{-5}$ at approximately $8.8 d B$ [9]. Both the ST-MLC and ST-LDPC code perform better than the Turbo code. The performance of all three approaches could be improved by using iterative decoding between the detection and decoding blocks as proposed in [9]. We believe the performance of the ST-MLC is better than that of the ST-LDPC code as it can calculate better soft information due to using $2 N_{t}$-D partitions and calculating the soft information using a basic signal set. In addition, the proposed ST-MLCs provide a good method of spreading information across space and time.

The ST-MLC uses a different detection method than the other two approaches, namely the ST-MSD. It is less complex than the approach used by the ST-LDPC code and Turbo code, as it takes advantage of the structure of the proposed ST-MLC.

\section{CONCLUSIONS}

We have developed a theoretical framework for the proposed ST-MLCs and ST-MSD. The proposed ST-MLC uses $2 N_{t^{-}}$ D rather than 2-D partitions. The partitioning strategy of [8] has been applied to the space-time environment, allowing the complexity of the resulting ST-MSD to be reduced. The proposed ST-MLC performed better than both the single level ST-LDPC code and Turbo code schemes.

\section{ACKNOWLEDGMENT}

The authors wish to thank the reviewers for their constructive criticism. This research was partially supported by the
Foundation for Research, Science and Technology of New Zealand.

\section{REFERENCES}

[1] V. Tarokh, N. Seshadri, and A. R. Calderbank, "Space-time codes for high data rate wireless communication: Performance criterion and code construction," IEEE Trans. Inform. Theory, vol. 44, pp. 744-765, Mar. 1998.

[2] G. Ungerboeck, "Channel coding with multilevel / phase signals," IEEE Trans. Inform. Theory, vol. 28, pp. 55-67, Jan. 1982.

[3] L. H.-J. Lampe, R. Schober, and R. F. H. Fischer, "Multilevel coding for multiple-antenna transmission," IEEE Trans. Wireless Commun., vol. 3, pp. 203-208, Jan. 2004.

[4] L. H.-J. Lampe, R. F. H. Fischer, and R. Schober, "Multilevel coding for multiple-antenna transmission," in Proc. ISIT, p. 104, Lausanne, Switzerland, June 30-July 52002.

[5] G. J. Pottie and D. P. Taylor, "Multilevel codes based on partitioning," IEEE Trans. Inform. Theory, vol. 35, pp. 87-98, Jan. 1989.

[6] D.-F. Yuan and X. Zhu, "Multiple hierarchical transmission scheme with bit interleaver over rayleigh fading channel," in Proc. VTC Fall, pp. 2439-2441, 7-11 Oct. 2001.

[7] D.-F. Yuan, F. Zhang, A.-F. Sui, and Z.-W. Li, "Concatenation of spacetime block codes and multilevel coding over rayleigh fading channels," in Proc. VTC Fall, pp. 192-196, 2001.

[8] L.-F. Wei, "Trellis-coded modulation with multidimensional constellations," IEEE Trans. Inform. Theory, vol. 33, pp. 483-501, July 1987.

[9] B. M. Hochwald and S. ten Brink, "Achieving near-capacity on a multiple-antenna channel," IEEE Trans. Commun., vol. 51, pp. 389-399, Mar. 2003.

[10] M. O. Damen, K. Abed-Meraim, and J.-C. Belfiore, "A generalized lattice decoder for asymmetrical space-time communication architecture," in Proc. ICASSP, pp. 2581-2584, 2000.

[11] P. A. Martin and D. P. Taylor, "High-throughput error correcting spacetime block codes," IEEE Commun. Lett., vol. 8, pp. 458-460, July 2004.

[12] P. A. Martin and D. P. Taylor, "High-throughput error correcting spacetime block codes," in Proc. ISIT, p. 127, Chicago, USA, June 27 - July 22004.

[13] J. Yuan, Z. Chen, B. Vucetic, and W. Firmanto, "Performance and design of space-time coding in fading channels," IEEE Trans. Commun., vol. 51, pp. 1991-1996, Dec. 2003.

[14] G. D. Forney Jr., "Coset codes - part 1: Introduction and geometrical classification," IEEE Trans. Inform. Theory, vol. 34, pp. 1123-1151, Sept. 1988.

[15] G. D. Forney Jr., "Coset codes - part 2: Binary lattices and related codes," IEEE Trans. Inform. Theory, vol. 34, pp. 1152-1187, Sept. 1988.

[16] S. Mallik and R. Koetter, "Partitioning techniques for design of multilevel codes for rayleigh fading channels," in Proc. ISIT, p. 409, Chicago, USA, June 27 - July 22004.

[17] S. Mallik, "Multilevel coding schemes for underspread fading channels," Master's thesis, University of Illinois at Urbana-Champaign, 2004.

[18] U. Wachsmann, R. F. H. Fischer, and J. B. Huber, "Multilevel codes: Theoretical concepts and practical design rules," IEEE Trans. Inform. Theory, vol. 45, pp. 1361-1391, July 1999.

[19] H. Zhu, Z. Shi, B. Farhang-Beroujeny, and C. Schlegel, "An efficient statistical approach for calculation of capacity of MIMO channels," in Proc. IASTED Int. Conf. Wireless Optical Commun., Bariff, Canada, July 14-16 2003.

[20] P. Robertson, E. Villebrun, and P. Hoeher, "A comparison of optimal and sub-optimal MAP decoding algorithms operating in the log domain," in Proc. ICC, vol. 2, pp. 1009-1013, 1995.

[21] T. Richardson, A. Shokrollahi, and R. Urbanke, "Design of capacityapproaching irregular low-density parity check codes," IEEE Trans. Inform. Theory, vol. 47, pp. 619-637, Feb. 2001.

[22] X.-Y. Hu, E. Eleftheriou, and D.-M. Arnold, "Progressive edge growth tanner graphs," in Proc. Globecom, vol. 2, pp. 995-1001, 2001.

[23] X.-Y. Hu, E. Eleftheriou, D.-M. Arnold, and A. Dholakia, "Efficient implementations of the sum-product algorithm for decoding LDPC codes," in Proc. Globecom, vol. 2, pp. 25-29, Nov. 2001.

[24] J. Boutros, N. Gresset, L. Brunel, and M. Fossorier, "Soft-input softoutput lattice sphere decoder for linear channels," in Proc. Globecom, San Francisco, CA, USA, 1-5 Dec. 2003.

[25] A. M. Chan and I. Lee, "A new reduced-complexity sphere decoder for multiple antenna systems," in Proc. ICC, pp. 460-464, 2002. 